\title{
CC chemokine ligand 7 expression in liver metastasis of colorectal cancer
}

\author{
YONG BEOM CHO ${ }^{1}$, WOO YONG LEE ${ }^{1}$, SO-JUNG $\mathrm{CHOI}^{2}$, JHINGOOK KIM ${ }^{2,3}$, \\ HYE KYUNG HONG ${ }^{1}$, SEOK-HYUNG KIM ${ }^{4}$, YOON-LA CHOI ${ }^{4}$, HEE CHEOL KIM ${ }^{1}$, \\ SEONG HYEON YUN ${ }^{1}$, HO-KYUNG CHUN ${ }^{1}$ and KUHN UK LEE ${ }^{5}$ \\ ${ }^{1}$ Department of Surgery, Samsung Medical Center, ${ }^{2}$ Samsung Biomedical Research Institute, \\ ${ }^{3}$ Department of Thoracic Surgery and ${ }^{4}$ Department of Pathology, Samsung Medical Center, Sungkyunkwan \\ University School of Medicine, Seoul 135-710; ${ }^{5}$ Department of Surgery, Seoul National University \\ College of Medicine, Seoul National University Hospital, Seoul 110-744, Republic of Korea
}

Received February 17, 2012; Accepted April 10, 2012

DOI: 10.3892/or.2012.1815

\begin{abstract}
The main cause of death for colorectal cancer (CRC) patients is the development of metastatic lesions at sites distant from the primary tumor. Therefore, it is important to find biomarkers that are related to the metastasis and to study the possible mechanisms. Recent data have shown that soluble attractant molecules called chemokines support the metastasis of certain cancers to certain organs. To identify molecular regulators that are differentially expressed in liver metastasis of CRC, PCR array analysis was performed and CC chemokine ligand 7 (CCL7) showed remarkable overexpression in liver metastatic tumor tissues. To validate the results of the PCR array, 30 patients with primary CRC and liver metastases were selected. Immunohistochemistry and real-time PCR analysis showed that CCL7 was expressed in normal colonic epithelium and the expression was higher in liver metastases compared to primary CRC $(\mathrm{P}<0.001)$. Real-time PCR showed that the expression of CCR1, CCR 2 and CCR3 was also higher in liver metastases compared to primary $\mathrm{CRC}(\mathrm{P}=0.001, \mathrm{P}=0.033$ and $\mathrm{P}<0.001$, respectively). In conclusion, correlation of CCL7 overexpression and its receptor expression with colon cancer liver metastasis suggests that CCL7 as a novel target in liver
\end{abstract}

Correspondence to: Dr Woo Yong Lee, Department of Surgery, Samsung Medical Center, Sungkyunkwan University School of Medicine, 50 Irwon-dong, Gangnam-gu, Seoul 135-710, Republic of Korea

E-mail: 1wy555@skku.edu

Dr Kuhn Uk Lee, Department of Surgery, Seoul National University College of Medicine, Seoul National University Hospital, 28 Yongon-dong, Chongno-gu, Seoul 110-744, Republic of Korea

E-mail:kulee@plaza.snu.ac.kr

Key words: colorectal cancer, liver metastasis, CC chemokine ligand 7 metastasis of CRC may be of potential clinical value for the prevention of hepatic recurrences.

\section{Introduction}

Colorectal cancer (CRC) is one of the three leading causes of cancer-related death among men and women worldwide. Although the 5-year survival rate for patients with localized CRC approaches $90 \%$, the spread of disease to distant sites decreases the 5 -year survival rate to $10 \%(1,2)$. The liver is the primary site of hematogenous metastases in colorectal cancer. Already at the time of diagnosis of the primary tumor, approximately $20 \%$ of patients present with synchronous liver metastases, and another $20-30 \%$ of patients will develop liver metastases after resection of the primary tumor (1). The cause of death for CRC patients is the development of metastatic lesions at sites distant from the primary tumor.

Tumor progression towards metastasis is often depicted as a multistage process in which malignant cells spread from the tumor of origin to colonize distant organs (3-5). Approximately a century ago, Paget (6) postulated in his 'seed and soil' hypothesis that successful interactions of tumor cells (seeds) with the microenvironment of a particular target organ (soil) leads to formation of distant metastases in specific organs. Cancer's predilection for selecting organs is likely explained by this theory that metastatic organs provide the optimal conditions for disseminated tumor cells to colonize, survive and proliferate.

Recent data have shown that soluble attractant molecules called chemokines support the metastasis of certain cancers to certain organs (7). The chemokines comprise a family of small basic chemotactic proteins whose effects are mediated by binding to G-protein-coupled receptors. They were originally identified by their ability to induce migration of leukocytes. Gradients of chemokines have been proposed to attract tumor cells with matching chemokine receptors to specific sites analogous to the directed homing of leukocytes (8-10). It is becoming increasingly clear that the chemokine network plays an important role in cancer through its effect on the growth 
Table I. Genes differentially expressed between primary and liver-metastatic CRC specimens.

\begin{tabular}{lllcr}
\hline Symbol & \multicolumn{1}{c}{ Gene name } & \multicolumn{1}{c}{ Description } & Average-fold change $^{\mathrm{a}}$ & P-value $^{-}$ \\
\hline CCL7 & FIC/MARC & Chemokine (C-C motif) ligand 7 & 9.26 & 0.0006 \\
FN1 & CIG/DKFZp686F10164 & Fibronectin 1 & 4.40 & 0.0039 \\
CXCR4 & CDI84/D2S201E & Chemokine (C-X-C motif) receptor 4 & 2.22 & 0.0421 \\
CST7 & CMAP & Cystatin F (leukocystatin) & 1.82 & 0.0491 \\
MGAT5 & GNT-V & Mannosyl $(\alpha-1,6-)$-glycoprotein & 1.77 & 0.0407 \\
& & $\beta-1,6-$ N-acetyl-glucosaminyltransferase & & \\
IL1B & IL-1/ILI- $\beta$ & Interleukin 1, $\beta$ & -6.18 & 0.0075 \\
MMP10 & SL-2/STMY2 & Matrix metallopeptidase 10 & -5.40 & 0.0105 \\
MMP2 & CLG4/CLG4A & Matrix metallopeptidase 2 & -4.07 & 0.0213 \\
MMP13 & CLG3 & Matrix metallopeptidase 13 & -4.58 & 0.0352 \\
CTSK & CTSO2/CTSO & Cathepsin K & -2.29 & 0.0434 \\
\hline
\end{tabular}

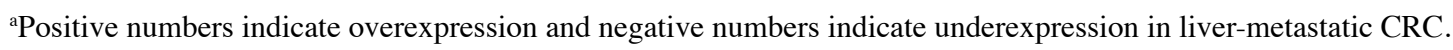

and metastasis of tumor cells as well as in manipulating hosttumor interactions (11).

$\mathrm{CC}$ chemokine ligand 7 (CCL7), also known as monocyte chemotactic protein-3 (MCP-3), is identified from osteosarcoma supernatant (12). It is expressed and secreted by monocytes, fibroblasts, platelets, colonic epithelial cells and some malignant tumor cells (13-15). By binding to CCR1, CCR2 and CCR3, CCL7 acts on the immune cells and activates monocytes, lymphocytes, dendritic cells, natural killer cells, and granulocytes $(16,17)$. There are evidence suggesting that CCL7 plays an important role in immune cells infiltrating in tumor cells and CCL7 gene transferred into tumor cells elicits antitumor effect such as reducing tumorigenicity and inhibiting tumor growth (17-20). However, limited data are available about the expression of CCL7 in CRC, and the role of CCL7 in liver metastasis of CRC has not been studied.

In this study, we used $\mathrm{RT}^{2}$ Profiler $^{\mathrm{TM}} \mathrm{PCR}$ array to identify genes that might play a significant role in liver metastasis in CRC and then we evaluated the expression of CCL7 and its receptors, CCR1, CCR2 and CCR3 in CRC-induced liver metastasis.

\section{Materials and methods}

Patient selection and tissue samples. Six primary colorectal carcinomas and their matched metastatic carcinoma from the liver were included in $\mathrm{RT}^{2}$ Profiler ${ }^{\mathrm{TM}}$ PCR array to identify possible target genes which show differential gene expression between primary and metastatic sites. All were synchronous cases in which primary and metastatic tumors were found at the same time. All tumors were fresh frozen specimens.

For validating study of CCL7, 30 patients with primary $\mathrm{CRC}$ and their liver metastases were selected. Patients' normal colon and liver tissues were selected to compare with the cancer tissues. Of the 30 primary and liver-metastatic CRCs, 17 were synchronous cases and 13 were metachronous cases in which metastatic tumors were found after surgery on primary tumors. Tissue samples were obtained from the archives of the Department of Pathology of the Samsung Medical Center.
Patients were selected from consecutively identified cases as long as their paraffin blocks were available. The investigation was approved by the Institutional Review Board (no. 200909-023).

$R T^{2}$ Profiler PCR array. Surgical specimens of six primary colorectal cancer tissues and their matched metastatic cancer to liver tissues were obtained and frozen at $-80^{\circ} \mathrm{C}$ until use. Selected frozen tissues were stained with $\mathrm{H} \& \mathrm{E}$ to improve visualization. Necrotic tumor tissues and intervening normal tissues were removed. Total RNAs were extracted from frozen tissues with Nucleospin RNA kit. cDNA was synthesized using an $\mathrm{RT}^{2}$ First Strand Synthesis kit (Super Array Bioscience, Frederick, MD, USA) and was analyzed using the Human tumor metastasis PCR array (Table I) and the $\mathrm{RT}^{2}$ SYBRGreen/Rox PCR Master Mix [APMM012C and PA-012-24, respectively (Super Array Bioscience)]. Data were normalized using multiple housekeeping genes and analyzed by comparing $2^{-\mathrm{ACt}}$ of the normalized sample. PCR was performed on ABI 7300 Real-Time PCR System (Applied Biosystems, Inc., Foster City, CA, USA).

Immunohistochemical staining and scoring. Immunohistochemical studies were carried out on formalin-fixed, paraffin-embedded, $4 \mu \mathrm{m}$-thick tissue sections. Rabbit antihuman polyclonal CCL7 antibody (GenWay Biotech, Inc., San Diego, CA, USA) was used at a dilution of 1:1000 for $30 \mathrm{~min}$. Tissue sections were deparaffinized three times in xylene for a total of $15 \mathrm{~min}$ and subsequently rehydrated. Antigen retrieval was carried out at $97^{\circ} \mathrm{C}$, PTLink (Dako, Glostrup, Denmark) for $20 \mathrm{~min}$ in citrate buffer ( $\mathrm{pH}$ 6.0) or EDTA buffer ( $\mathrm{pH}$ 8.0). Then, immunostaining was performed using Bond Max autoimmunostainer (Leica Biosystems, Melbourne, Australia). Briefly, after blocking the endogenous peroxidase activity with $3 \%$ hydrogen peroxidase for $5 \mathrm{~min}$, the primary antibody incubation was carried out for $15 \mathrm{~min}$. The antigen-antibody reaction was detected using Bond ${ }^{\mathrm{TM}}$ Polymer Refine Detection, DS9800 (Vision BioSystems, Melbourne, Australia). Counterstaining was performed with Mayer's hematoxylin. 
CCL7 expressions were estimated using both staining intensity and proportion. Intensity was scored as follows: 0 , no staining; 1 , weak staining; 2 , moderate staining; and 3, strong staining. Proportion was scored as follows: 0 , no staining; 1 , positive area between $1-25 \%$; 2 , positive area between $26-50 \%$; 3 , positive area between $51-75 \%$; and 4 , positive area between $76-100 \%$. Quantitative analyses of the CCL7 expressions were determined by immunoreactivity score which was determined as intensity score multiplied by proportion score. Cancer cell and stroma were graded each apart.

Quantitative real-time RT-PCR for CCL7, CCR1, CCR2 and CCR3 mRNA expression. Total RNA was extracted from paraffin blocks using MasterPure ${ }^{\mathrm{TM}}$ Complete DNA and RNA Rurification kit (Epicentre Biotechnologies) according to the manufacturer's instructions. Amplification of mRNA was performed and then it was transcribed from doublestranded cDNA using SuperScript ${ }^{\mathrm{TM}}$ III Reverse transciptase (Invitrogen).

Quantitative real-time RT PCR was performed in triplicate in 384-well plates; each $10 \mu \mathrm{l}$ reaction consisted of $5 \mu 1$ of Power SYBR ${ }^{\circledR}$-Green PCR Master Mix (Applied Biosystems), $0.25 \mu \mathrm{l}$ of $10 \mu \mathrm{M}$ concentrated Primer, Probe sets of CCL7 (Bioneer Oligo Synthesis Report), CCR1 (Bioneer Oligo Synthesis Report), CCR2 (Bioneer Oligo Synthesis Report), CCR3 (Bioneer Oligo Synthesis Report) and GAPDH (Bioneer Oligo Synthesis Report). CCL7 primers were: sense 5'-TGCT CAGCCAGTTGGGATTA-3' and antisense 5'-GGACAGTG GCTACTGGTGGT'3'. CCR1 primers were: sense 5'-CTGG T TGGAAACATCCTGGT-3' and antisense 5'-GGAAGCGTG AACAGGAAGAG-3'. CCR2 primers were: sense 5'-CCCCA GTCACCTGCTGTTAT-3' and antisense 5'-GCTTCTTTGG GACACTTGCT-3'. CCR3 primers were: sense 5'-GTGTTC ACTGTGGGCCTCTT-3' and antisense 5'-GTGACGAGGA AGAGCAGGTC-3'. GAPDH primers were: sense 5'-GCACC GTCAAGGCTGAGAA-3' and antisense 5'-AGCATCGCCC CACTTGATT-3'. The real-time PCR analysis was performed on an Applied Biosystems Prism 7900 Sequence Detection System (Applied Biosystems).

Statistical analysis. Ct values were normalized for the deviations against the average of five housekeeping genes: $B 2 M$, RPRT1, HPRT1, GAPDH and ACTB. The differential gene expression was estimated as: $\Delta \mathrm{Ct}=\mathrm{Ct}_{\text {(liver metastases) }}-\mathrm{Ct}_{\text {(colon }}$ primary, and fold-change $=2^{(-\Delta \mathrm{Ct})}$. Quantitative analysis of the array data on the primary colon and the metastatic liver tumors was carried out by the Web-based PCR array data analysis software provided by the manufacturer (http://www. sabiosciences.com).

The Volcano plot was plotted according to the values of fold-change and P-value between primary and liver-metastatic tumor. The Volcano plot considered genes that had $\geq 4$-fold change and a P-value $<0.05$ in the t-test was selected.

Statistical analyses were conducted to compare CCL7, CCR1, CCR 2 and CCR3 mRNA expression levels between primary CRC and liver-metastatic carcinoma using the paired t-test or Wilcoxon signed ranks test. P-value of $<0.05$ was considered statistically significant. PASW statistical software version 17 (SPSS Inc., Chicago, IL, USA) was used for all statistical analyses.

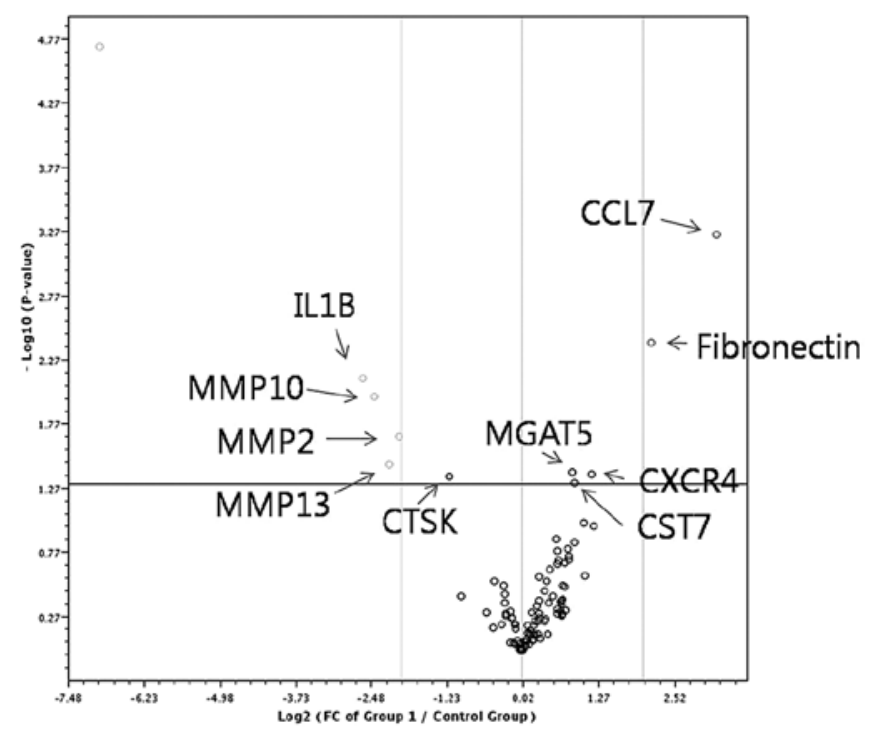

Figure 1. Volcano plot of metastasis PCR array in 6 paired primary and livermetastatic colorectal carcinoma. Group 1 is for the metastatic liver tissues and control group is for the primary colorectal tissues. Data analysis was performed using the $\Delta \Delta \mathrm{Ct}$ method with normalization of the raw data to either housekeeping genes or an external RNA control.

\section{Results}

Identification of differentially expressed genes between primary and liver-metastatic CRC using $R T^{2}$ Profiler PCR array. The mean expressions of CCL7, fibronectin, CXCR4, CST7 and MGAT5 were significantly higher in liver-metastatic tumor, whereas those of IL1B, MMP10, MMP2, MMP13 and CTSK were lower compared with the primary tumor (Table I). When metastatic tumor was compared with primary tumor, Volcano plot analysis generated a gene list of 6 genes, of which 2 genes were upregulated and 4 genes were downregulated (Fig. 1). There was a remarkable upregulation of CCL7 in liver-metastatic tumor tissues (average fold-change, 9.26). Therefore, CCL7 was selected for validation of the following studies.

Expression of CCL7. We observed the CCL7 expression in normal colon and liver tissues (Fig. 2). Immunohistochemical staining revealed that CCL7 was expressed in the cytoplasm of normal colonic epithelial cells, fibroblasts and inflammatory cells, but CCL7 was not expressed in the normal hepatocytes.

We compared the CCL7 expression in primary CRC with its matched liver-metastatic cancer. The immunohistochemical staining revealed that CCL7 is expressed in both primary and liver-metastatic cancer tissues (Fig. 3A). The CCL7 positive staining was located mostly in epithelial and fibromuscular stromal cells. Quantitative analysis using the immunoreactivity score showed that levels of CCL7 expression of liver-metastatic tissues were greater than those of primary CRC tissues in both cancer cell and stroma (Fig. 3B, P $<0.001$ ). These results demonstrated that CCL7 protein was more highly expressed in most liver-metastatic tissues compared to primary CRC tissues suggesting that CCL7 may play a critical role in liver metastasis of CRC. 

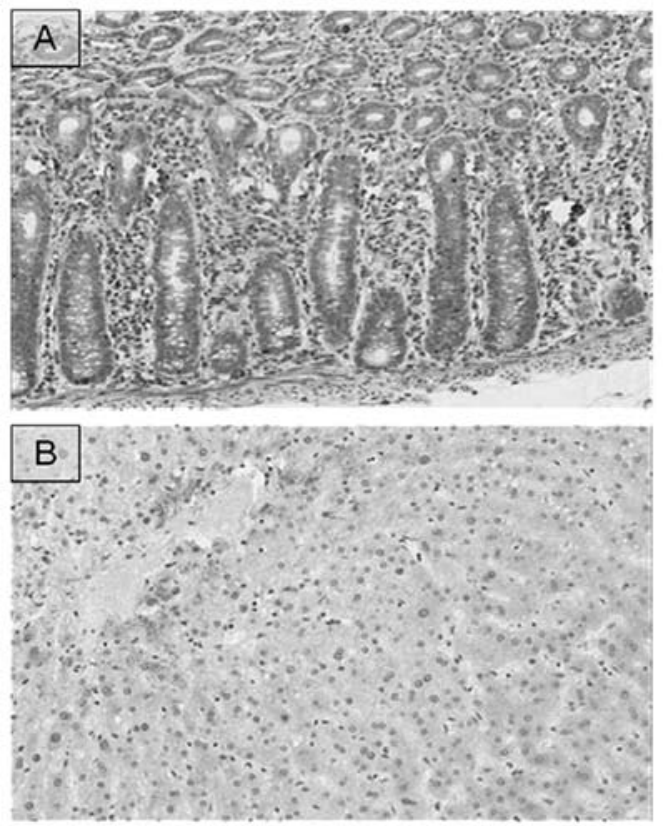

Figure 2. The immunohistochemical staining of CCL7 in normal colon and liver tissues. (A) CCL7 was expressed in the cytoplasm of normal colonic epithelium, stromal fibroblasts and inflammatory cells (original magnification, $\mathrm{x} 200$ ). (B) There was no CCL7 expression in normal hepatocytes (original magnification, $\mathrm{x} 200$ ).
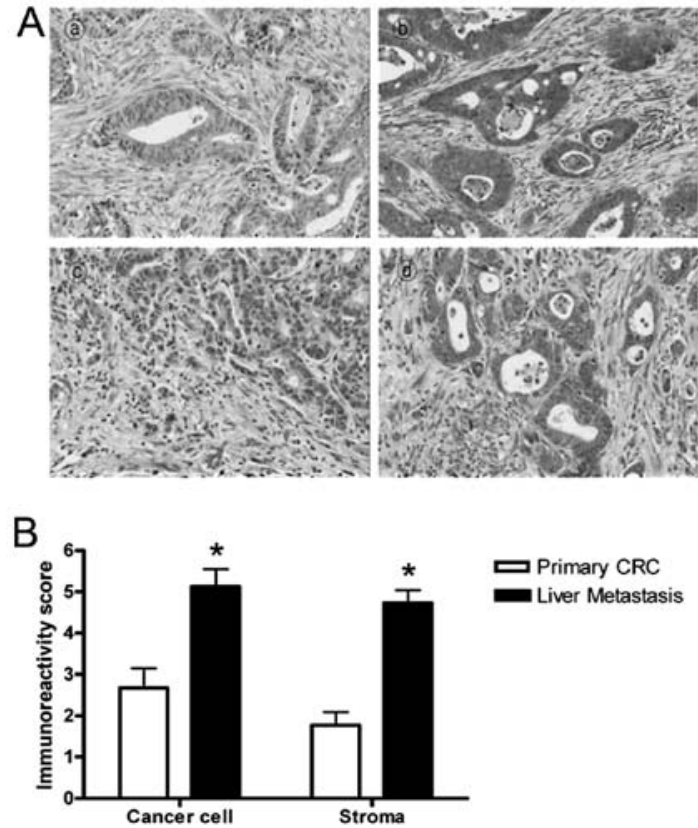

Figure 3. CCL7 expression and immunoreactivity score in primary and livermetastatic tumor tissues. (A) Immunohistochemical staining of primary CRC ( $\mathrm{a}$ and $\mathrm{b}$ ) and liver metastasis ( $\mathrm{c}$ and $\mathrm{d}$ ) using the polyclonal antibody for CCL7. Original magnification of $x 200$ is shown. (a and c) Low expression of CCL7. (b and d) High expression of CCL7. (B) Quantitative evaluation of CCL7 expression using the immunoreactivity score. The mean immunoreactivity score \pm SD are presented in a graphical format using error bar with $95 \%$ confidence intervals. Statistically significant differences were noted between primary and liver-metastatic cancer tissues in both cancer cells and stroma $(\mathrm{P}<0.001)$.

Quantitative real-time RT-PCR was used to verify the expression of CCL7 mRNA in primary CRC tissues and liver-

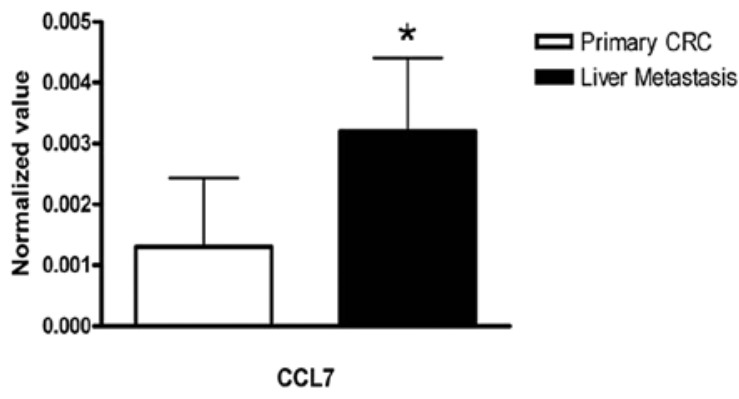

Figure 4. The mRNA expression of $C C L 7$ in the primary and liver-metastatic CRC tissue by using real-time RT-PCR. The expression levels of $C C L 7$ mRNA in each specimen were detected by real-time RT-PCR. Three independent experiments were performed. Results are presented as means \pm SD of these independent experiments. " $\mathrm{P}<0.001$ vs. primary CRC.

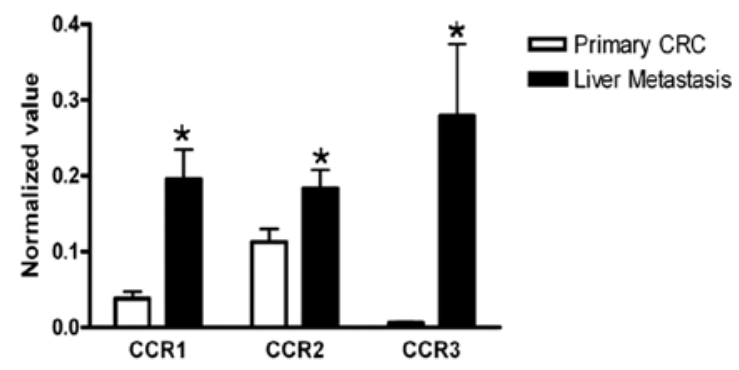

Figure 5. The mRNA expression of $C C R 1, C C R 2$ and $C C R 3$ in the primary and liver-metastatic CRC tissue by using real-time RT-PCR. Expression of $C C R 1, C C R 2$ and $C C R 3$ in the primary CRC and their corresponding liver metastasis tissue were detected by real-time PCR. Three independent experiments were performed. Results are presented as means \pm SD of these independent experiments. ${ }^{*} \mathrm{P}<0.05$ vs. primary CRC.

metastatic tissues. CCL7 mRNA expression was significantly higher in liver-metastatic tissues than in primary CRC tissues (Fig. 4, $\mathrm{P}<0.001$ ).

Expression of CCR1, CCR2 and CCR3. Because CCL7 has been known to act through specific receptors, CCR1, CCR2 and CCR3, we investigated the expression of CCR1,CCR2 and CCR3. We analyzed the mRNA expressions of CCR1, CCR2, and $C C R 3$ in the primary CRC tissues and their corresponding liver-metastatic tissue samples by using real-time RT-PCR. The results revealed that all the expressions of $C C R 1, C C R 2$ and $C C R 3$ in liver-metastatic tissues were significantly higher than those in the corresponding primary CRC tissues (Fig. 5, $\mathrm{P}=0.001, \mathrm{P}=0.033$, and $\mathrm{P}<0.001$, respectively).

\section{Discussion}

In this study, we performed $\mathrm{RT}^{2}$ Profiler PCR array to identify biomarkers expressed differentially between primary CRC and liver metastasis and then $C C L 7$ was selected as a possible biomarker related to the liver metastasis of CRC. In order to evaluate the possible relation of CCL7 with liver metastasis of CRC, we analyzed expression levels of CCL7 and its receptors CCR1, CCR2, and CCR3 in 30 primary CRC specimens with their corresponding liver metastasis tissues. The results from real-time RT-PCR analysis showed that mRNA expressions of both $C C L 7$ and its receptors $C C R 1, C C R 2$ and $C C R 3$ were 
higher in liver metastasis tissues than those in their corresponding primary CRC tissues. By immunohistochemical staining, we found that CCL7 was expressed in the normal colonic epithelium, colon cancer cells, and liver-metastatic cells, but not in normal hepatocytes.

A mechanism most recently ascribed to organ-specific cancer metastasis is the paradigm of chemokine-mediated cell migration. Since chemokine signaling results in the directional migration and specific arrival of cells at a target destination, chemokines have emerged as crucial molecules in the metastatic process. From the initial studies by Muller et al (7), numerous articles have been published regarding chemokine/chemokine receptor interactions with metastasis. Various studies implicate chemokines CXCL12, CCL20, CCL19, and CCL21 and their corresponding receptors in the tumorigenic process and metastatic homing of tumor cells. An association between the CCL20 and its receptor CCR6 expression in the colorectal cancer progression and liver metastasis is previously reported, suggesting involvement of CCL20/CCR6 chemokine receptor pair in the promotion of colorectal cancer liver metastasis (21-23). In recent years the CC-chemokines CCL2 and CCL7 attracted considerable interest as inflammatory mediators that play pleiotropic tumorigenic roles in breast cancer homing to bone and metastases growth through osteoblast induction (24). A recent study demonstrated that CCL2 expressed by both metastatic breast cancer tumors and stroma plays a critical role in tumor cell extravasation and metastatic seeding that is mediated via inflammatory monocyte recruitment (25). Morrison et al (26) showed that cleavage of CCL2 and CCL7 by MMP-13 generates forms of the chemokines that are potent receptor antagonists in a breast cancer bone metastasis model. Jung et al (27) reported that CCL7 promoted the invasion and migration of oral squamous cell carcinoma cells (OSCC), and the invasiveness was inhibited by treatment with CCL7 neutralizing antibody. They also demonstrated that inhibiting CCR1 and CCR3 reduces CCL7-induced OSCC cell migration, suggesting that CCL7 promotes cancer cell migration through those receptors in OSCC cells. These data prompted us to comparatively investigate CCL7 and its receptors expression profiles in liver metastases in colorectal cancer. In this study, we observed significantly higher CCL7 expression in the liver metastases tissues compared with their corresponding primary CRC tissues. Furthermore, CCL7 receptors, CCR1, CCR2 and CCR3, were also overexpressed in the liver metastases suggest that the malignant status of a colorectal cancer cell might be correlated with CCL7 and its receptors expression.

Since the identification of chemokines as key targets in cancer metastasis has emerged as research topic, the investigation of the CCL7 as novel target in liver metastasis of CRC may be of potential clinical value for the prevention of hepatic recurrences. Therefore, further studies to investigate the functions of CCL7 in liver metastasis of CRC and the mechanism of interaction between CCL7 and its receptors CCR1, CCR2, and CCR3 should be conducted.

\section{Acknowledgements}

This research was supported by the Basic Science Research Program through the National Research Foundation of
Korea (NRF) funded by the Ministry of Education, Science and Technology (no. 2010-0025652), a grant of the Korea Healthcare technology R\&D Project, Ministry for Health and Welfare Affairs, Republic of Korea (A092255), Samsung Biomedical Research Institute grant (\#SBRI C-B0-318-1) and grants from IN-SUNG Foundation for Medical Research (C-A9-852-1).

\section{References}

1. Jemal A, Murray T, Ward E, Samuels A, Tiwari RC, Ghafoor A, Feuer EJ and Thun MJ: Cancer statistics, 2005. CA Cancer J Clin 55: 10-30, 2005.

2. Kim J, Takeuchi H, Lam ST, Turner RR, Wang HJ, Kuo C, Foshag L, Bilchik AJ and Hoon DS: Chemokine receptor CXCR4 expression in colorectal cancer patients increases the risk for recurrence and for poor survival. J Clin Oncol 23: 2744-2753, 2005.

3. Christofori G: New signals from the invasive front. Nature 441: 444-450, 2006.

4. Gupta GP and Massague J: Cancer metastasis: building a framework. Cell 127: 679-695, 2006.

5. Steeg PS: Tumor metastasis: mechanistic insights and clinical challenges. Nat Med 12: 895-904, 2006.

6. Paget S: The distribution of secondary growths in cancer of the breast. 1889. Cancer Metastasis Rev 8: 98-101, 1989.

7. Muller A, Homey B, Soto H, Ge N, Catron D, Buchanan ME, McClanahan T, Murphy E, Yuan W, Wagner SN, Barrera JL, et al: Involvement of chemokine receptors in breast cancer metastasis. Nature 410: 50-56, 2001.

8. Zlotnik A and Yoshie O: Chemokines: a new classification system and their role in immunity. Immunity 12: 121-127, 2000.

9. Zlotnik A: Chemokines and cancer. Int J Cancer 119: 2026-2029, 2006.

10. Lee JH, Cho YS, Lee JY, Kook MC, Park JW, Nam BH and Bae JM: The chemokine receptor CCR4 is expressed and associated with a poor prognosis in patients with gastric cancer. Ann Surg 249: 933-941, 2009.

11. Balkwill F: Cancer and the chemokine network. Nat Rev Cancer 4: 540-550, 2004.

12. Van Damme J, Proost P, Lenaerts JP and Opdenakker G: Structural and functional identification of two human, tumorderived monocyte chemotactic proteins (MCP-2 and MCP-3) belonging to the chemokine family. J Exp Med 176: 59-65, 1992.

13. Uguccioni M, D'Apuzzo M, Loetscher M, Dewald B and Baggiolini M: Actions of the chemotactic cytokines MCP-1, MCP-2, MCP-3, RANTES, MIP-1 alpha and MIP-1 beta on human monocytes. Eur J Immunol 25: 64-68, 1995.

14. Menten P, Proost P, Struyf S, Van Coillie E, Put W, Lenaerts JP, Conings R, Jaspar JM, De Groote D, Billiau A, et al: Differential induction of monocyte chemotactic protein-3 in mononuclear leukocytes and fibroblasts by interferon-alpha/beta and interferon-gamma reveals MCP-3 heterogeneity. Eur J Immunol 29: 678-685, 1999.

15. Power CA, Clemetson JM, Clemetson KJ and Wells TN: Chemokine and chemokine receptor mRNA expression in human platelets. Cytokine 7: 479-482, 1995.

16. Xu LL, McVicar DW, Ben-Baruch A, Kuhns DB, Johnston J, Oppenheim JJ and Wang JM: Monocyte chemotactic protein-3 (MCP3) interacts with multiple leukocyte receptors: binding and signaling of MCP3 through shared as well as unique receptors on monocytes and neutrophils. Eur J Immunol 25: 2612-2617, 1995

17. Hu JY, Li GC, Wang WM, Zhu JG, Li YF, Zhou GH and Sun QB: Transfection of colorectal cancer cells with chemokine MCP-3 (monocyte chemotactic protein-3) gene retards tumor growth and inhibits tumor metastasis. World J Gastroenterol 8: 1067-1072, 2002.

18. Wang JM, Deng X, Gong W and Su S: Chemokines and their role in tumor growth and metastasis. J Immunol Methods 220: 1-17, 1998.

19. Fujita M, Furukawa Y, Nagasawa Y, Ogawa M and Nakamura Y: Down-regulation of monocyte chemotactic protein-3 by activated beta-catenin. Cancer Res 60: 6683-6687, 2000. 
20. Fioretti F, Fradelizi D, Stoppacciaro A, Ramponi S, Ruco L, Minty A, Sozzani S, Garlanda C, Vecchi A and Mantovani A: Reduced tumorigenicity and augmented leukocyte infiltration after monocyte chemotactic protein-3 (MCP-3) gene transfer: perivascular accumulation of dendritic cells in peritumoral tissue and neutrophil recruitment within the tumor. J Immunol 161: 342-346, 1998.

21. Rubie C, Oliveira V, Kempf K, Wagner M, Tilton B, Rau B, Kruse B, Konig $\mathbf{J}$ and Schilling $\mathbf{M}$ : Involvement of chemokine receptor CCR6 in colorectal cancer metastasis. Tumour Biol 27: 166-174, 2006.

22. Ghadjar P, Coupland SE, Na IK, Noutsias M, Letsch A, Stroux A, Bauer S, Buhr HJ, Thiel E, Scheibenbogen C and Keilholz U: Chemokine receptor CCR6 expression level and liver metastases in colorectal cancer. J Clin Oncol 24: 1910-1916, 2006.

23. Rubie C, Frick VO, Wagner M, Weber C, Kruse B, Kempf K, Konig J, Rau B and Schilling M: Chemokine expression in hepatocellular carcinoma versus colorectal liver metastases. World J Gastroenterol 12: 6627-6633, 2006.
24. Bar-Shavit Z: The osteoclast: a multinucleated, hematopoieticorigin, bone-resorbing osteoimmune cell. J Cell Biochem 102: 1130-1139, 2007

25. Qian BZ, Li J, Zhang H, Kitamura T, Zhang J, Campion LR, Kaiser EA, Snyder LA and Pollard JW: CCL2 recruits inflammatory monocytes to facilitate breast-tumour metastasis. Nature 475: 222-225, 2011

26. Morrison C, Mancini S, Cipollone J, Kappelhoff R, Roskelley C and Overall C: Microarray and proteomic analysis of breast cancer cell and osteoblast co-cultures: role of osteoblast matrix metalloproteinase (MMP)-13 in bone metastasis. J Biol Chem 286: 34271-34285, 2011

27. Jung DW, Che ZM, Kim J, Kim K, Kim KY, Williams D and Kim J: Tumor-stromal crosstalk in invasion of oral squamous cell carcinoma: a pivotal role of CCL7. Int J Cancer 127: 332-344, 2010. 